p. 452; the first two lines of Lemma 2.4 should read: "If $a_{m+1}(x), \ldots, a_{m+p}(x)$ are fixed $(\bmod v)$, then one can find for each $i(1 \leqslant i \leqslant k)$ exactly one $j(i) \leqslant k$ such that $x(m+p) \in S_{i}^{\prime}$ is equivalent".

p. 452, line 6 from bottom should start with " $\geqslant \varepsilon h P\{\ldots$ ".

p. 453, line 9 should have at the end "(take $a_{1}=1$ and $\left.r=1\right)$ ".

p. 454, line 4 from bottom should read

$$
\Delta_{m, p} \leqslant \lambda_{5} \Delta_{m+M+n, p-M-n}+O_{5} \lambda_{5}^{n} .
$$

p. 460 , lines 2,3 from bottom should read " $\left(\Sigma^{\prime \prime}\right.$ is over $k$ from $I$ to $a_{m+1}(x)$ but includes only those $k$ with $\left.k q_{m} \leqslant N\right)^{\prime \prime}$.

p. 464, formula (3.19) should be: $\frac{q_{8}(x)}{q_{n}(x)} \frac{1}{\left(q_{n}(x)+q_{n-1}(x)\right)}$.

p. 464, line 2 from bottom $a_{1}(x), \ldots, a_{m+1}(x)$ should be $a_{1}(x), \ldots, a_{m+2}(x)$

p. 466, line $3 A(\eta) \exp (-(m-p) \eta)$ should be $A(\eta) \exp (-(m-p) B(\eta))$.

p. 469 , line $7 C_{8} \frac{t}{\log N}$ should be $-C_{8} \frac{|t|}{\log N}$.

p. 469 , formula (3.31). The sum over $m$ should run from $m=2 p$ to $m=(\tau-\varepsilon) \log N$. This requires corresponding changes on p. 470 .

\title{
References
}

[1] W. Doeblin, Remarques sur la theorie métrique des fractions continues, Comp. Math. 7 (1939-40), pp. 353-371.

[2] J, L. Doob, Asymptotic properties of Markoff transition probabilities, Trans, Am. Math. Soc. 63 (1948), pp. 393-421.

[3] G. H. Hardy and E. M. Wright, An introduction to number theory, Ch. 10, 3rd edition, Oxford, 1954.

[4] Harry Kesten, Uniform distribution mod1, Ann. of Math. 71 (1960), pp. 445.471

[5] A. Khintchine, Kettenbrüche, Part C, Leipzig, 1956.

[6] B. O. Koopman and J. v. Neumann, Dynamical systems of continuous spectra, Proc. Nat. Acad. of Sci. 18 (1932), pp. 255-263.

[7] Paul Lévy, Théorie de l'addition des variables aléatories, Cl. 9, 2nd ed., Paris, 1954.

[8] P. Szüsz, Über einen Kusminschen Satz, Acta Math. Hung. 12 (1961), pp. 447 453

[9] H. Weyl, Über die Gleichverteilung von Zahlen mod Eins, Math. Ann. 77 (1916), pp. 313-352.

CORNELL UNIVERSITY

ITHACA, NEW YORE

Reçu par la Rédaction le 6. 2. 1962

\section{Binomial coefficients in an algebraic number field *}

by

L. CARLitz (Durham, N. C.)

1. Let $K=R(\Theta)$ denote an algebraic number field of degree $n$ over the rationals. Let $\mathfrak{p}$ be a prime ideal of $K$ and let $p$ be the rational prime divisible by $p$. Let $K_{\mathfrak{p}}$ denote the set of numbers of $K$ that are integral $(\bmod p)$. Put

$$
\left(\begin{array}{l}
a \\
m
\end{array}\right)=\frac{\alpha(\alpha-1) \ldots(\alpha-m+1)}{m !} .
$$

We shall prove the following result.

THEOREM 1. The binomial coefficients $\left(\begin{array}{l}a \\ m\end{array}\right)$ are integral (modp) for all $\alpha \in K_{\mathfrak{p}}$ and all $m \geqslant 1$ if and only if $\mathfrak{p}$ is a prime ideal of the first degree and moreover $p$ does not divide the discriminant of $K$.

Proof. To prove the necessity of the stated conditions suppose first that $\mathfrak{p}$ is of degree $f>1$. Then the residue class ring $K_{\mathfrak{p}} / \mathfrak{p}$ is a finite field of order $p^{f}$. Since $f>1$ there exists a number $\alpha \in K_{p}$ such that

$$
\alpha \neq \equiv(\operatorname{modp}) \quad(r=0,1, \ldots, p-1) .
$$

Therefore the binomial coefficient $\left(\begin{array}{l}\alpha \\ p\end{array}\right)$ is not integral (modp).

Next let $p$ be of the first degree but let $p$ divide the discriminant of $K$. Then by Dedekind's theorem on discriminantal divisors, $\mathfrak{p}^{2} \mid p$. Also there exists an integer $\alpha$ of $K$ such that ([3], p. 97, Theorem 74]

$$
(a, p)=\dot{\mathfrak{p}} \text {. }
$$

Since $p$ is of the first degree, the numbers

$$
a, a-1, \ldots, \alpha-p+1
$$

constitute a complete residue system $(\operatorname{modp})$. Clearly only the first of these numbers is divisible by $\mathfrak{p}$. Therefore by (1.1) the product

$$
\alpha(\alpha-1) \ldots(\alpha-p+1)
$$

* Supported in part by National Science Foundation grant G 16485. 
is divisible by $\mathfrak{p}$ but not by $\mathfrak{p}^{2}$. It follows that $\left(\begin{array}{l}a \\ p\end{array}\right)$ is not integral (modp). This completes the proof of the necessity.

To prove the sufficiency, assume that $p$ is of the first degree and that $p^{2} \nmid p$. Then for $r \geqslant 1$ the numbers

$$
0,1,2, \ldots, p^{r}-1
$$

form a complete residue system $\left(\bmod p^{r}\right)$. For if two are congruent $\left(\bmod \mathfrak{p}^{r}\right)$ we should have $p^{r} \mid t$, where $1 \leqslant t<p^{r}$. If $p^{*}$ is the highest power of $p$ dividing $t$ it follows that $p^{r} \mid p^{s}$; since $p^{2}+p$ we get $r \leqslant s$ which is evidently impossible.

If $a$ is an arbitrary number of $K_{\mathfrak{p}}$ it follows from the above that the numbers

$$
\alpha, \alpha-1, \ldots, \alpha-p^{r}+1
$$

constitute a complete residue system $\left(\operatorname{modp} p^{r}\right)$. Thus in the sequence

$$
\alpha, \alpha-1, \ldots, \alpha-m+1
$$

there are $[\mathrm{m} / \mathrm{p}]$ multiples of $\mathfrak{p},\left[\mathrm{m} / \mathrm{p}^{2}\right]$ multiples of $\mathrm{p}^{2}$, and so on. Therefore the product

is divisible by $\mathfrak{p}^{w}$, where

$$
-\alpha(\alpha-1) \ldots(\alpha-m+1)
$$

$$
w=\left[\frac{m}{p}\right]+\left[\frac{m}{p^{2}}\right]+\ldots
$$

Since $m$ ! is divisible by exactly $p^{w}$ and therefore by exactly $p^{w}$, it follows that $\left(\begin{array}{l}a \\ m\end{array}\right)$ is integral (modp).

As a corollary of Theorem 1 we have

THEOREM 2. Let $p$ be a rational prime and let $K_{p}$ denote the set of numbers of $K$ that are integral $(\bmod p)$. Then the binomial coefficients $\left(\begin{array}{l}a \\ m\end{array}\right)$ are integral $(\bmod p)$ for all $\alpha \in K_{p}$ and all $m \geqslant 1$ if and only if

$$
(p)=\mathfrak{p}_{1} \mathfrak{p}_{2} \ldots \mathfrak{p}_{n}
$$

where the $\mathfrak{p}_{j}$ are distinct prime ideals (of the first degree) of $K$.

To prove the theorem let

$$
(p)=\mathfrak{p}_{1}^{e_{1}} \ldots \mathfrak{p}_{r}^{e_{r}}
$$

be the prime ideal decomposition of $(p)$ in $K$, where the $p_{j}$ are distinct prime ideals. Then by Theorem $1,\left(\begin{array}{l}a \\ m\end{array}\right)$ is integral $\left(\bmod \mathfrak{p}_{j}\right)$ for all $a \in K_{\mathfrak{p}_{j}}$ and all $m \geqslant 1$ if and only if $\mathfrak{p}_{j}$ is of the first degree and $e_{j}=1$. Since $K_{p}$ is the intersection of all $K_{\mathfrak{p},}$ it.follows that $\left(\begin{array}{l}\alpha \\ m\end{array}\right)$ is integral for all $a \in K_{p}$ and all $m \geqslant 1$ if and only if all $\mathfrak{p}_{j}$ are of the first degree and all $e_{j}=1$. Thus (1.3) reduces to (1.2).

For a special case of Theorem 2 see [1], p. 586, Lemma.

2. As in Theorem 1 let $p$ be a prime ideal of the first degree such that $p \nmid p$. We shall determine the residue $(\bmod p)$ of $\left(\begin{array}{l}a \\ m\end{array}\right)$. Since, as we have seen above, the numbers

$$
0,1,2, \ldots, p^{N}-1
$$

constitute a complete residue system $\left(\bmod p^{N}\right)$ we may put

$$
a=c_{0}+c_{1} p+\ldots+c_{N-1} p^{N-1}\left(\bmod \mathfrak{p}^{N}\right),
$$

where the $c_{j}$ are rational integers, $0 \leqslant c_{j}<p$. Put

$(2.2)$

so that by (2.1)

$$
c=c_{0}+c_{1} p+\ldots+c_{N-1} p^{N-1},
$$

$$
\text { It follows from (2.3) that }
$$

$$
\text { (2.4) } \quad \alpha(\alpha-1) \ldots(\alpha-m+1) \equiv c(c-1) \ldots(c-m+1)\left(\operatorname{modp} p^{N}\right),
$$

where $m$ is an arbitrary integer $\geqslant 1$. Put

Then (2.4) implies

$$
\begin{gathered}
m=m_{0}+m_{1} p+\ldots+m_{r-1} p^{r-1} \quad\left(0 \leqslant m_{j}<p\right) \\
\nu(m)=\left[\frac{m}{p}\right]+\left[\frac{m}{p^{2}}\right]+\ldots+\left[\frac{m}{p^{r-1}}\right] .
\end{gathered}
$$

$$
\left(\begin{array}{l}
a \\
m
\end{array}\right) \equiv\left(\begin{array}{l}
c \\
m
\end{array}\right)\left(\bmod p^{N-r(m)}\right) \text {. }
$$

We now suppose that $N>v(m)$ and recall the theorem due to Lucas ([2], p. 271) that, in the present notation,

$$
\left(\begin{array}{c}
c \\
m
\end{array}\right) \equiv\left(\begin{array}{c}
c_{0} \\
m_{0}
\end{array}\right)\left(\begin{array}{c}
c_{1} \\
m_{1}
\end{array}\right) \cdots\left(\begin{array}{c}
c_{r-1} \\
m_{r-1}
\end{array}\right)(\bmod p) .
$$

It should be observed that if in (2.1) $N$ is replaced by $N+1$, the coefficients $c_{0}, c_{1}, \ldots, c_{N-1}$ do not change. We therefore get from (2.5) and (2.6)

We may state

$$
\left(\begin{array}{c}
a \\
m
\end{array}\right) \equiv\left(\begin{array}{c}
c_{0} \\
m_{0}
\end{array}\right)\left(\begin{array}{c}
c_{1} \\
m_{1}
\end{array}\right) \cdots\left(\begin{array}{c}
c_{r+1} \\
m_{r-1}
\end{array}\right)(\operatorname{modp}) .
$$

THEOREM 3. Let $\mathfrak{p}$ be a prime ideal of the first degree such that $\mathfrak{p}^{2} \nmid p$. If

and we put

$$
m=m_{0}+m_{1} p+\ldots+m_{r-1} p^{r-1} \quad\left(0 \leqslant m_{j}<p\right)
$$

$$
a \equiv c_{0}+c_{1} p+\ldots+c_{r-1} p^{r-1}(\bmod p r) \quad\left(0 \leqslant c_{j}<p\right),
$$


where $a$ is an arbitrary number $\in K_{p}$, then (2.7) holds. In particular $\left(\begin{array}{l}a \\ m\end{array}\right)$ is prime to $\mathfrak{p}$ if and only if
(2.10)
$m_{j} \leqslant c_{j} \quad(j=0,1, \ldots, r-1)$.

As a corollary we have the following result supplementary to Theorem 2 .

THEOREM 4. Let

$$
(p)=p_{1} p_{2} \ldots p_{n},
$$

where the $\mathfrak{p}_{j}$, are distinct prime ideals of the first degree of $K$. Also let (2.11) $\quad \alpha \equiv c_{k 0}+c_{k 1} p+\ldots+c_{k, r-1} p^{r-1}\left(\bmod \mathfrak{p}_{k}^{r}\right) \quad\left(0 \leqslant c_{k j}<p\right)$.

Then $\left(\begin{array}{l}a \\ m\end{array}\right)$ is prime to $p$ if and only if

$$
m_{j} \leqslant \min _{1<k<n} c_{k j} \quad(j=0,1, \ldots, r-1),
$$

where

$$
m=m_{0}+m_{1} p+\ldots+m_{r-1} p^{r-1} \quad\left(0 \leqslant m_{j}<p\right) .
$$

3. It is evident from the proof of Theorem 3 that if $\alpha=\beta\left(\bmod p^{r}\right)$ then

$$
\left(\begin{array}{l}
a \\
m
\end{array}\right) \equiv\left(\begin{array}{c}
\beta \\
m
\end{array}\right)(\bmod p)
$$

provided $m<p^{r}$. To get a more general result we require the following

LEMIMA. Let $a, b$ be rational integers such that

Then

$$
a \equiv b\left(\bmod p^{r+s}\right) \quad(r \geqslant 1, s \geqslant 0) .
$$

$$
\left(\begin{array}{l}
a \\
m
\end{array}\right) \equiv\left(\begin{array}{c}
b \\
m
\end{array}\right)\left(\bmod p^{s+1}\right) \quad\left(1 \leqslant m<p^{r}\right) .
$$

Proof. Put $a=b+c$ and consider

$$
(1+x)^{a}=(1+x)^{b}(1+x)^{c} .
$$

Clearly (3.2) is an immediate consequence of

(3.3) $\quad\left(\begin{array}{c}c \\ m\end{array}\right) \equiv 0\left(\bmod p^{s+1}\right) \quad\left(1<m<p^{r}\right)$.

Since

$$
\left(\begin{array}{l}
c \\
m
\end{array}\right)=\frac{c}{m}\left(\begin{array}{c}
n-1 \\
m-1
\end{array}\right)
$$

and

$$
\frac{c}{m} \equiv 0\left(\bmod p^{s+1}\right) \quad\left(1 \leqslant m<p^{r}\right),
$$

(3.3) follows at once.

Now let $\alpha, \beta \in K_{\mathfrak{p}}$, where $\mathfrak{p}$ is of the first degree and $\mathfrak{p}^{2}+p$. Then we may put

$$
\begin{aligned}
& \alpha \equiv a_{0}+a_{1} p+\ldots+a_{N-1} p^{N-1}\left(\bmod p^{N}\right), \\
& \beta \equiv b_{0}+b_{1} p+\ldots+b_{N-1} p^{N-1}\left(\bmod p^{N}\right),
\end{aligned}
$$

where $0 \leqslant a_{j}<p, 0 \leqslant b_{j}<p$ and $N$ is at our disposal. We assume that

$$
\alpha \equiv \beta\left(\bmod p^{r+s}\right)
$$

and take $N>r+s$. It follows that

Put

$$
a_{j}=b_{j} \quad(0 \leqslant j<r+s) .
$$

so that

$$
\begin{gathered}
a=a_{0}+a_{1} p+\ldots+a_{N-1} p^{N-1}, \\
b=b_{0}+b_{1} p+\ldots+b_{N-1} p^{N-1}, \\
c=a_{0}+a_{1} p+\ldots+a_{r+s-1} p^{r+s-1}, \\
a \equiv a, \quad \beta \equiv b\left(\operatorname{modp} p^{N}\right), \\
a \equiv b \equiv c\left(\bmod p^{r+s}\right) .
\end{gathered}
$$

For sufficiently large $N$, it follows from (3.5) that

$$
\left(\begin{array}{l}
a \\
m
\end{array}\right) \equiv\left(\begin{array}{l}
a \\
m
\end{array}\right), \quad\left(\begin{array}{l}
\beta \\
m
\end{array}\right) \equiv\left(\begin{array}{l}
b \\
m
\end{array}\right)\left(\bmod p^{s+1}\right) .
$$

On the other hand, it follows from (3.6) and the Lemma that

$$
\left(\begin{array}{l}
a \\
m
\end{array}\right) \equiv\left(\begin{array}{l}
b \\
m
\end{array}\right)\left(\bmod p^{s+1}\right) \text {. }
$$

Combining (3.7) and (3.8) we get

\section{This proves}

$$
\left(\begin{array}{c}
a \\
m
\end{array}\right) \equiv\left(\begin{array}{l}
\beta \\
m
\end{array}\right)\left(\bmod p^{s+1}\right) \text {. }
$$

THEOREM 5. Let $\mathfrak{p}$ be a prime ideal of the first degree such that $\mathfrak{p}^{2}+p$. Let $a, \beta$ be numbers of $K_{\mathfrak{p}}$ such that

where $r \geqslant 1, s \geqslant 0$. Then

$$
\alpha \equiv \beta\left(\operatorname{modp} p^{r+s}\right),
$$

$$
\left(\begin{array}{l}
\alpha \\
m
\end{array}\right) \equiv\left(\begin{array}{l}
\beta \\
m
\end{array}\right)\left(\bmod p^{s+1}\right)
$$

for all $m<p^{r}$.

THEOREM 6. Let

$$
(p)=\mathfrak{p}_{1} p_{2} \ldots p_{n}
$$

where the $\mathfrak{p}_{j}$ are distinct prime ideals of the first degree of $K$. Let $\alpha, \beta$ be numbers of $K_{p}$ such that

where $r \geqslant 1, s \geqslant 0$. Then

$$
\alpha \equiv \beta\left(\bmod p^{r+s}\right)
$$

for all $m<p^{r}$.

$$
\left(\begin{array}{l}
a \\
m
\end{array}\right) \equiv\left(\begin{array}{c}
\beta \\
m
\end{array}\right)\left(\bmod p^{s+1}\right)
$$


4. If again $\mathfrak{p}$ is of the first degree and $p^{2}+p$ we can determine the highest power of $p$ dividing $\left(\begin{array}{l}a \\ m\end{array}\right)$ in the following way. Put

$$
m=m_{0}+m_{1} p+\ldots+m_{r-1} p^{r-1} \quad\left(0 \leqslant m_{j}<p\right)
$$

and let $\alpha \equiv a\left(\operatorname{modp} p^{N}\right)$, where

$$
a=a_{0}+a_{1} p+\ldots+a_{N^{N-1}} p^{N-1} \quad\left(0 \leqslant a_{j}<p\right) .
$$

For $N$ sufficiently large it is clear that $\left(\begin{array}{l}\alpha \\ m\end{array}\right)$ and $\left(\begin{array}{l}a \\ m\end{array}\right)$ are divisible by the same powers of $p$; moreover for $\left(\begin{array}{l}a \\ m\end{array}\right)$ this is the same as the highest power of $p$ dividing $\left(\begin{array}{l}a \\ m\end{array}\right)$.

Now by Kummer's rule ([2], p. 270) the highest power of $p$ dividing

where

$$
\left(\begin{array}{c}
b+c \\
c
\end{array}\right) \quad(b \geqslant 0, c \geqslant 0)
$$

$$
\begin{array}{ll}
b=b_{0}+b_{1} p+\ldots+b_{s} p^{s} & \left(0 \leqslant b_{j}<p\right), \\
c=c_{0}+c_{1} p+\ldots+c_{s} p^{s} & \left(0 \leqslant c_{j}<p\right),
\end{array}
$$

is determined as follows. Let

$$
\begin{aligned}
b_{0}+c_{0} & =a_{0}+e_{0} p, \\
b_{1}+c_{1}+e_{0} & =a_{1}+e_{1} p, \\
\cdots \ldots+\cdots & \cdots \\
b_{s-1}+c_{s-1}+e_{s-2} & =a_{s-1}+e_{s-1} p, \\
b_{s}+e_{s}+e_{s-1} & =a_{s}+e_{s} p,
\end{aligned}
$$

where each $e=0$ or 1 . Then $\left(\begin{array}{c}b+c \\ b\end{array}\right)$ is divisible by exactly $p^{e}$, where

We now put

$$
e=e_{0}+e_{1}+\ldots+e_{s} \text {. }
$$

so that

$$
a^{(k)}=a_{0}+a_{1} p+\ldots+a_{k} p^{k} \quad(k=0,1,2, \ldots),
$$

and apply Kummer's rule to

$$
\left(\begin{array}{c}
a^{(k)} \\
m
\end{array}\right) \quad(k=r, r+1, \ldots) .
$$

We assume that $m \leqslant a^{(r)}$. It follows that all the binomial coefficients (4.3) are divisible by exactly the same power of $p$, for clearly there is no additional "earrying" when $k>r$.

This proves

THEOREM 7 . Let $p$ be a prime ideal of the first degree such that $p^{2} \nmid p$ and let

$$
\begin{array}{cc}
m=m_{0}+m_{1} p+\ldots+m_{r-1} p^{r-1} & \left(0 \leqslant m_{j}<p\right), \\
\alpha \equiv a_{0}+a_{1} p+\ldots+a_{r} p^{r}\left(\bmod p^{r-1}\right) & \left(0 \leqslant a_{j}<p\right) .
\end{array}
$$

Moreover assume that

$$
m \leqslant a=a_{0}+a_{1} p+\ldots+a_{r} \cdot p^{r} .
$$

Then the highest power of $\mathfrak{p}$ dividing $\left(\begin{array}{l}a \\ m\end{array}\right)$ is the same as the highest power of $p$ dividing $\left(\begin{array}{l}a \\ m\end{array}\right)$. The latter power can be found by means of Kummer's rule (4.1).

THEOREM 8. Let

$$
(p)=\mathfrak{p}_{1} \mathfrak{p}_{2} \ldots \mathfrak{p}_{n},
$$

where the $p_{j}$ are distinct prime ideals of the first degree. Let

$$
m=m_{0}+m_{1} p+\ldots+m_{r-1} p^{r-1} \quad\left(0 \leqslant m_{j}<p\right),
$$

$\alpha \equiv c_{k} \equiv c_{k 01}+c_{k 1} p+\ldots+c_{k, r} p^{r}\left(\bmod p_{k}^{r+1}\right) \quad\left(0 \leqslant c_{k j}<p ; k=1, \ldots, n\right)$.

Let $p^{e_{k}}$ denote the highest power of $p$ dividing $\left(\begin{array}{l}c_{k} \\ m\end{array}\right)$ and assume that

$$
m \leqslant \min \left(c_{1}, \ldots, c_{k}\right) .
$$

Then the binomial coefficient $\left(\begin{array}{l}a \\ m\end{array}\right)$ is divisible by exactly $p^{e}$, where

$$
e=\min \left(e_{1}, \ldots, e_{k}\right) \text {. }
$$

Remark. The hypothesis $m \leqslant a$ occurring in Theorem 7 is necessary for the application of Kummer's rule. A like remark applies to the hypothesis

in Theorem 8.

$$
m \leqslant \min \left(c_{1}, \ldots, c_{n}\right)
$$

As a corollary of the last two theorems we have

THEOREM 9. Let $p$ be a prime ideal of the first degree such that $\mathfrak{p}^{2}+p$ and let $m<p^{r}$. Then if

where

$$
\alpha \equiv \beta \equiv a\left(\bmod \mathfrak{p}^{r+1}\right),
$$

$$
a=a_{0}+a_{1} p+\ldots+a_{r} p^{r} \quad\left(0 \leqslant a_{j}<p\right)
$$

and in addition $m \leqslant a$ it follows that $\left(\begin{array}{c}a \\ m\end{array}\right)$ and $\left(\begin{array}{c}\beta \\ m\end{array}\right)$ are divisible by exactly the same power of $\mathrm{p}$.

$$
\text { If moreover } \quad(p)=p_{1} p_{2} \ldots p_{n},
$$

where the $\mathrm{p}_{j}$ are distinct prime ideals of the first degree, then $\left(\begin{array}{l}\alpha \\ m\end{array}\right)$ and $\left(\begin{array}{l}\beta \\ m_{i}\end{array}\right)$ are divisible by exactly the same power of $p$.

Remark. As in Theorems 7 and 8 the condition $m \leqslant a$ is necessary for Kummer's rule. 
[1] L. Carlitz, Some arithmetic properties of a special sequence of polynomials, Duke Math. Journ. 26 (1959), pp. 583-590.

[2] L. E. Dickson, History of the theory of numbers, vol. 1, Washington 1919.

[3] E. Hecke, Theorie der algebraischen Zahlen, Leipzig 1923.

\section{Solvability of certain equations in a finite field*}

by

\author{
L. CARLTTZ (Durham, N. C.)
}

1. Let $q=p^{n}$, where $p$ is a prime, and let $G F(q)$ denote the finite field of order $q$. Schwarz [4] has given an elegant proof of the following theorem. If $k \mid p-1$, if $a_{1}, \ldots, a_{k}$ are non-zero numbers of $G F(q)$ and $a$ is an arbitrary number of the field, then the equation

$$
a_{1} x_{1}^{k}+\ldots+a_{k} x_{k}^{k}=a
$$

has at least one solution in the field.

Using the same method, the writer [2] has proved the following theorems.

THEOREM 1. Let $k \mid p-1$ and let $a_{1}, \ldots, a_{k}$ be non-zero numbers of $G F(q)$. Let $g\left(x_{1}, \ldots, x_{k}\right)$ be an arbitrary polynomial with coefficients in $G F(q)$ of degree less than $k$. Then the equation

$$
a_{1} x_{1}^{k}+\ldots+a_{k} x_{k}^{k}=g\left(x_{1}, \ldots, x_{k}\right)
$$

has at least one solution in the field.

THEOREM 2. If $f\left(x_{1}, \ldots, x_{k}\right)$ is homogeneous of degree $k$ while $g\left(x_{1}, \ldots, x_{k}\right)$ is arbitrary of degree less than $k$, and

then the equation

$$
\sum_{x_{1}, \ldots, x_{k} \in G F^{\prime}(\alpha)}\left\{f\left(x_{1}, \ldots, x_{k}\right)\right\}^{q-1} \neq 0
$$

$$
f\left(x_{1}, \ldots, x_{k}\right)=g\left(x_{1}, \ldots, x_{k}\right)
$$

has at least one solution in the field. Alternatively the condition (1.1) may be replaced by the equivalent statement that the number of solutions of the equation

$$
f\left(x_{1}, \ldots, x_{k}\right)=0
$$

is not divisible by $p$.

By the degree of $g\left(x_{1}, \ldots, x_{k}\right)$ is understood the total degree.

* Supported in part by National Science Foundation grant G 16485.

Acta Arithmetica VII 